\title{
Temporal response of magnitude distribution to fluid injection rates in The Geysers geothermal field
}

\author{
Konstantinos Leptokaropoulos ${ }^{1} \cdot$ Monika Staszek $^{1}$
}

Received: 3 April 2018 / Accepted: 20 October 2018 / Published online: 30 October 2018

(c) The Author(s) 2018

\begin{abstract}
The influence of fluid injection rates on the magnitude distribution of the seismicity which occurred in the NW part of The Geysers geothermal site is studied here. A direct comparison between injection rate changes and $b$ value response is attempted after appropriate selection of data subsets. Due to the relatively small sample (1121 events, corresponding to an average rate of $\sim 0.45$ events/day), we also aggregated seismic activity into two families corresponding to increasing and decreasing injection rates, respectively. The $b$ values were calculated as a function of time lag related to the injection activity. In agreement with previous studies, we found a statistically significant direct relation between $b$ values and injection rate changes, which occurred at a zero or very short time lag (from 0 to $\sim 15$ days). However, the $b$ value changes are related to the slope (i.e., the second derivative of injection volume), instead of the absolute values of injection rates. The increasing injection rates correspond to $b=1.18 \pm 0.06$, whereas the decreasing injection rates correspond to $b=1.10 \pm 0.05$. The corresponding values estimated by the repeated medians technique are $b=1.97 \pm 0.20$ and $b=1.50 \pm 0.13$. Both differences are significant at 0.05 level.
\end{abstract}

Keywords Injection-induced seismicity $\cdot$ Magnitude distribution $\cdot$ Geothermal fields $\cdot$ Statistical seismology

\section{Introduction}

The high demands for ores and energy in addition to the recent requirement for better usage of the underground resources have resulted in an enhancement of the anthropogenic seismicity in urbanized and industrialized areas worldwide (e.g., Grigoli et al. 2017 and references therein). Remarkable seismic activity is recorded in areas, which were up to date characterized by low (if any) seismicity and negligible hazard levels. Some of these anthropogenic events are strong enough to be felt on the surface or cause damage to the operational infrastructure. It has been particularly shown in many previous studies that fluid injection into reservoirs, connected with activities such as hydro-fracking for unconventional oil and gas extraction or geothermal system stimulation, frequently induces seismicity. (See Davies et al. 2013; Zang et al. 2014 for review.) Following its definition, hydraulic fracturing aims at creating new cracks and

Konstantinos Leptokaropoulos

kleptoka@igf.edu.pl

1 Institute of Geophysics, Polish Academy of Sciences, Warsaw, Poland enhancing permeability. For this purpose, fracking operations involve injection of fluid into a well for achieving a sustainable exploitation of oil, gas or geothermal energy. When fluid pressure is efficiently high (higher than minimum principal stress), it leads to fracture of the rock mass, inducing tensile microseismicity in the surrounding area. More important is the fact that an increase in the pore pressure which takes place during injection also results in the frictional resistance reduction in preexisting faults (effective normal stress concept, Terzaghi 1943), which consequently can lead to release of the accumulated stress in the form of seismic events (e.g., Langenbruch and Zoback 2016).

The aforementioned mechanism, evident at injection sites and especially in enhanced geothermal fields (e.g., Majer et al. 2007), results in specific characteristics of induced seismicity which may significantly differ from the tectonic earthquakes. One of the most prominent features of injection-induced seismicity is its frequency-magnitude distribution. The Gutenberg-Richter (G-R) law's $b$ value, indicating the relative frequency of small to large events, is generally higher in the case of induced seismicity (1.2-2.0, occasionally higher) than tectonically originated one $(\sim 1.0)$ (e.g., Wyss 1972; Dempsey et al. 2016). For example, Bachmann 
et al. (2012) found an average $b \sim 1.3$ for the Basel-enhanced geothermal system, which substantially decreased from the co-injection period $\left(b_{\mathrm{co}}=1.57 \pm 0.06\right)$ to the post-injection period $\left(b_{\text {post }}=1.14 \pm 0.06\right)$. There are cases, however, where $b$ values of induced events are comparable or even lower than the ones characterizing natural seismicity (e.g., Huang and Beroza 2015; Goebel et al. 2016). Moreover, events caused directly by hydraulic fracturing often demonstrate a $b$ value $\sim 2.0$, whereas fault reactivation events have a lower $b$ value 1.0 (e.g., Maxwell et al. 2009; Davies et al. 2013). It is also worth noticing that there are cases when injection operations only trigger negligible seismic activity above the detection level of surface networks (e.g., Evans et al. 2012; López-Comino et al. 2018). $b$ values are also highly variable, generally decreasing systematically with time and distance from the open hole of the injection well (e.g., Bachmann et al. 2012). Although the observations are often contradictory with each other depending on particular case studies and geological features, in general, high injection rates lead to increased $b$ values and vice versa. However, estimating the spatial and temporal changes in magnitude distribution of induced events is not a trivial task, due to the large samples required for a robust $b$ value evaluation and the small spatial and temporal scales in which these changes may occur.

Several physical mechanisms have been proposed in order to interpret field and laboratory observations on $b$ value variation. The most commonly assumed concept is the uncoupled approach, according to which the elastic stresses and pore pressure are independent, i.e., changes in pore pressure act equally on all three normal stresses and therefore the differential stress remains unaffected. In such case, the system is moved toward failure (Mohr's circle shifted to the left) at lower stress accumulation, and thus, the strain energy is released by smaller rather that larger events (Bachmann et al. 2012; El-Isa and Eaton 2014 and references therein), sometimes with significantly lower stress drops (Staszek et al. 2017). However, several recent studies imply stress-pore pressure coupling (e.g., Hillis 2003; Schoenball et al. 2010) which may result in a differential stress decrease during injection rate increase and vice versa. Goertz-Allmann and Wiemer (2013) developed and applied a geomechanical model for geothermal reservoirs and found that the higher the pore pressure change, the lower the differential stress becomes. This, in turn, leads to an inverse relation between differential stress and $b$ value as shown by Scholz (2015). Therefore, both coupled and uncoupled approaches should lead to an increase in $b$ value at increasing injection rates. Nevertheless, such relation is not always obviously revealed from observational data due to the relatively large uncertainties and the inherent complexity of the process.

Our objective is to seek for a relation between magnitude distribution and operational data (fluid injection) fluctuation in the NW of The Geysers geothermal field (California, USA). For this purpose, we perform a statistical data analysis to segmental as well as aggregated seismic and operational data for quantifying the significance of the correlation between $b$ value and injection rate variation. We analyze $b$ values calculated in the proximity of Prati-9 and Prati-29 injection wells from seismic events which occurred during (1) periods of increasing injection rate and (2) periods of decreasing injection rate. Then, we quantify the correlation between $b$ values and injection rates and seek for a potential time delay of maximum and statistically significant correlation. The problem of $b$ value estimation uncertainty is addressed by deploying independent methods of $b$ value determination and by calculating the corresponding standard errors by means of bootstrap resampling. Finally, we check whether any relation between $b$ value and absolute injection rate values, spatial microseismicity distribution, and static stress drop can be observed.

\section{Data}

The Geysers is a vapor-dominated geothermal field constantly operating in California since 1960s. Nowadays, the steam production is enhanced by low-pressure water injections: Cool water falls freely into the reservoir via injection wells leading to high temperature contrast between the fluid and reservoir. The Geysers, in general, experienced a very low level of seismic activity prior to the onset of power production. Due to the small number of stations installed before the development of the geothermal resource, background seismicity data are limited. Although very sparse historical seismicity has been evident prior to 1960 (e.g., Oppenheimer 1986), the geothermal operations clearly modulate the seismic activity since then: Induced thermal stresses and pore pressure fluctuations lead to the occurrence of numerous seismic events in the entire area (see Majer and Peterson 2007; Martínez-Garzón et al. 2014).

For the purpose of this study, we used an isolated cluster of seismicity located in the northwestern part of The Geysers geothermal field in the close vicinity of Prati-9 and Prati-29 injection wells (Fig. 1 from Martínez-Garzón et al. 2014). This cluster contains 1254 events which took place between December 10, 2007, and August 23, 2014. These events were relocated from the original NCEDC catalog by Kwiatek et al. (2015), and the resulting catalog demonstrates an average relative hypocentral error of less than $50 \mathrm{~m}$. In this study, we focused on the main cluster consisting of 1121 events which forms a distinct cloud located at the maximum distance of up to $600 \mathrm{~m}$ from Prati-9 (Fig. 1a). The events magnitudes were recalculated from NCEDC catalog by Kwiatek et al. (2015) using the formula $M_{\mathrm{W}}=0.9 M_{\mathrm{D}}+0.47$ proposed by Edwards and Douglas (2014). The complete 
Fig. 1 a Cross section with the spatial distribution of analyzed seismicity cloud: black dots represent 1121 events which were used for $b$ value estimation, blue dots are remaining 133 events belonging to the original dataset. Red crosses assign open holes of Prati-9 and Prati-29 injection wells. b Moment magnitude distribution of the analyzed dataset. c Total and individual injection rates into Prati-9 and Prati-29 injection wells. The vertical shaded bars indicate the periods selected for $b$ value estimation in our study: blue-correspond to increasing injection rate, pink to decreasing injection rate and gray to approximately constant injection rate. The red bar indicates the time period discarded from our analysis due to the anomalously high corresponding $b$ value
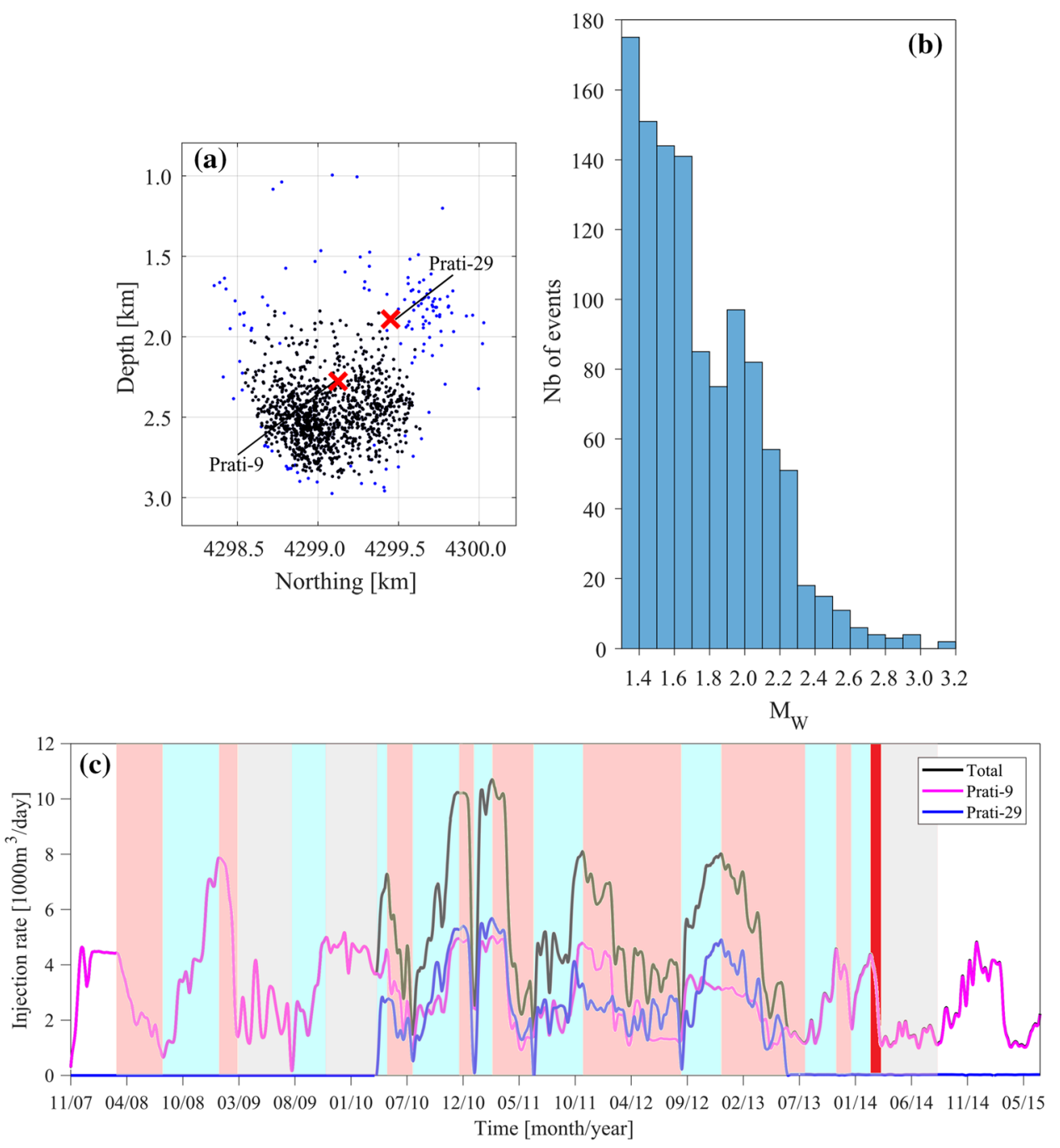

dataset includes events with magnitudes from 1.37 to 3.16 (Fig. 1b), and the magnitude $\left(M_{\mathrm{W}}=1.37\right)$ of completeness was estimated by Kwiatek et al. (2015) applying the $95 \%$ goodness-of-fit test (Wiemer and Wyss 2000). The same authors found that $\mathrm{M}_{\mathrm{C}}$ fluctuates between 1.37 and $\sim 1.60$, as estimated in 200-event windows; however, estimating completeness level for small subsets may lead to artifacts resulting from limited data number (Leptokaropoulos et al. 2018a). For this reason, we followed the approach of Kwiatek et al. (2015) and Martínez-Garzón et al. (2018), which assumes a uniform $M_{\mathrm{C}}$ for the entire dataset. Static stress drops (SD) were calculated for 322 events, among 1121 chosen for the analysis, by Kwiatek et al. (2015) using mesh spectral ratio technique. Six events which occurred before February 1, 2010, were discarded from the dataset due to seismic network reconstruction (see Staszek et al. 2017), so the final stress drop dataset used consisted of 316 events.

Injection activity into Prati-9 well was continuous during the entire analyzed time period, whereas injection into
Prati-29 initiated in April 2010 and was carried on until June 2013 (Martínez-Garzón et al. 2014; Kwiatek et al. 2015; IS EPOS, 2017). In both wells, injection had seasonal character with peak injection rates taking place during winter months (Fig. 1c).

\section{Methodology}

A variety of parametric and nonparametric approaches have been proposed and routinely applied for describing the magnitude distribution of both tectonic and anthropogenic seismicity (e.g., Kijko and Sellevoll 1989; Utsu 1999; Urban et al. 2016; Leptokaropoulos et al. 2017). The most commonly used approach assumes that the magnitudes are independent and identically distributed random variables with an infinitely large possible maximum magnitude. This assumption leads to the so-called unbounded G-R distribution of magnitudes for $M \geq M_{\min }$, where $M_{\min }$ is the completeness magnitude of the 
analyzed dataset. To estimate $b$ value in this study, we applied the maximum likelihood estimator of Aki (1965) as follows:

$\hat{b}=\frac{1}{\ln (10)\left[\langle M\rangle-\left(M_{\min }-\Delta M / 2\right)\right]}$

where $\langle M\rangle$ stands for the sample mean of the dataset and $\Delta \mathrm{M}$ represents the values binning (round-off interval) of the reported magnitudes (Bender 1983). Aki (1965) also provided the estimator of $\hat{b}$ standard deviation, $\sigma_{\mathrm{b}}$, defined as:

$\sigma_{\mathrm{b}}=\frac{\hat{b}}{\sqrt{N}}$

where $N$ stands for the sample size. The asymptotic distribution of $\hat{b}$ is normal $\left(N\left(\hat{b}, \sigma_{b}\right)\right)$ and $\sigma_{\mathrm{b}}$ can be evaluated by Eq. (2), adequately allowing for a robust estimation of the $b$ value confidence intervals given relatively large samples. We therefore made use of this estimator when the datasets contained more than 150 samples, whereas for smaller datasets, we performed bootstrap confidence intervals evaluation. For that purpose, 10,000 realizations of random samples drawn randomly with replacement were performed for each dataset. The confidence intervals were determined corresponding to the 0.16 and 0.84 quantiles, indicating one standard deviation assuming normally distributed random samples. (This assumption can be considered as valid based on the large number of realizations applied.) The same bootstrap resampling procedure was followed to estimate the standard deviation of the injection rates in selected time periods, as described in the next section.

In order to further investigate the statistical significance of $b$ value difference, we applied an alternative $b$ value estimation technique, as proposed by Amorèse et al. (2010). The applied method is a nonparametric regression approach, namely the Siegel (1982) repeated medians technique which is considered to be highly resistant to outliers and large observational uncertainties, also requiring limited a priori information on the errors (Smirnov 2003; Amorèse et al. 2010). The associated methodology which was developed for the repeated medians $b$ value calculation and the bootstrap estimate of the corresponding standard error is described in detail in Amorèse et al. (2010). For each calculation step, 10,000 bootstrap resamplings with replacement were realized in our study, and the magnitude bin size was selected equal to 0.1 and 0.01 units. We sought for statistical significance of the $b$ values variation corresponding to periods of increasing and periods of decreasing injection rate slope, which corresponds to the derivative of injection rate (or, equivalently, the second derivative of injection volume). The significance of this difference in the estimated $b$ values was determined by a bootstrap $t$ test (Amorèse et al. 2010): $t=\frac{\left|b_{\text {increasing }}^{\mathrm{RM}}-b_{\text {decreasing }}^{\mathrm{RM}}\right|}{\sqrt{S E_{\text {increasing }}^{2}+S E_{\text {decreasing }}^{2}}}$

where $b_{\text {increasing }}^{\mathrm{RM}}$ and $b_{\text {decreasing }}^{\mathrm{RM}}$ are the $b$ values estimated by the repeated medians technique, and $S E_{\text {increasing }}$ and $S E_{\text {decreasing }}$ are the bootstrap standard errors of each $b$ value estimates (arising from 10,000 resamplings each). If $t>\sim 1.96$, then the difference is significant at 0.05 level.

\section{Results}

For the purpose of this study, various types of $b$ value analysis were performed in order to investigate thoroughly the impact of changes of fluid injection operations on magnitude distribution. A crucial part of the analysis is the division of the original dataset into smaller ones corresponding to different injection activity intensities. The original injection data reports daily injection rates. Those rates were averaged for each one of the time windows considered and their standard deviations were estimated by bootstrap resampling. As seen in Fig. 1c, the injection rates exhibit intense fluctuations at different time scales. Short-term fluctuations (i.e., $<\sim 1$ month) were discarded from the analysis for two reasons: (1) In most of the cases, their amplitudes are negligible in comparison with the long-term trend of injection rates, and (2) their short duration corresponds to extremely limited number (if any) of available events. In both cases, the statistical analysis leads to ambiguous results and high uncertainties. For these reasons, we selected manually 21 time periods of relatively constant injection rate slope ( 9 periods with increasing, 9 with decreasing, and 3 with average unchanged injection rates-Fig. 1c). The seismic events which occurred within the duration of each one of the aforementioned time periods were used for $b$ value estimation and comparison with the fluid injection rate slope. This approach was followed in order to investigate whether a long-term increase or decrease in the injection rate influences the magnitude distribution of the induced events. A similar technique was applied by Leptokaropoulos et al. (2018b); however, their datasets considered only strictly monotonically increasing or decreasing injection rates, to account for even minor fluctuations of injection operations. Here, we generalize this concept to the long-term injection rate trend in order to include more data and obtain more robust results. Most of the calculated $b$ values for each one of the 21 aforementioned periods are found between 0.80 and 1.45 ; however, there is a period of decreasing injection rate which contains only 18 events and exhibits an anomalously high $b=3.04$. This value is 
considered as an outlier, and therefore, it was discarded from the following analysis. (See Appendix Fig. 9) After that, 20 datasets remained: nine corresponding to periods of increasing, eight to periods of decreasing, and three to periods of stable injection rates. A positive linear correlation between the slope (or gradient) of the injection rate and $b$ values is shown in Fig. 2. Although some variations are evident, it is clear that higher $b$ values are estimated for periods of increasing injection rates and lower $b$ values for periods of decreasing injection rates. The Spearman rank correlation coefficient (SCC) between the two variables is equal to 0.59 with a corresponding $p=0.007$, indicating that the correlation is significant at 0.01 level.

Nevertheless, due to the limited sample, it is not feasible to achieve robust results for subsets (e.g., separately for increasing or decreasing rates). As a result of the data limitation, the derived uncertainties are large especially in the $b$ value estimation, shown by the error bars in Fig. 2 . This led us to follow an different approach for verifying and strengthening these findings by enhancing the stability and significance of the statistical analysis results. In the previously described approach, the 20 periods tested provide an adequate sample of injection rate cycle; however, these periods contain a limited number of events (from 20 to 155 events). This fact leads to large uncertainties in the $b$ value estimation. Leptokaropoulos et al. (2018a) showed that sample size inherently influences the magnitude distribution parameters, such that in small samples, the $b$ value is generally overestimated. In our case, the data sample sizes, although small, are comparable with each other, and therefore, the uncertainties are comparable as well in majority of cases. However, in order to provide more robust $b$ value estimation, we aggregated the datasets in two families: one corresponding to periods of increasing injection rate (440 events) and one corresponding to periods of decreasing injection rate (492 events). In such way, the size of the data to compare was efficiently increased (by a factor of 3-25, compared to the individual datasets). The analysis indicates

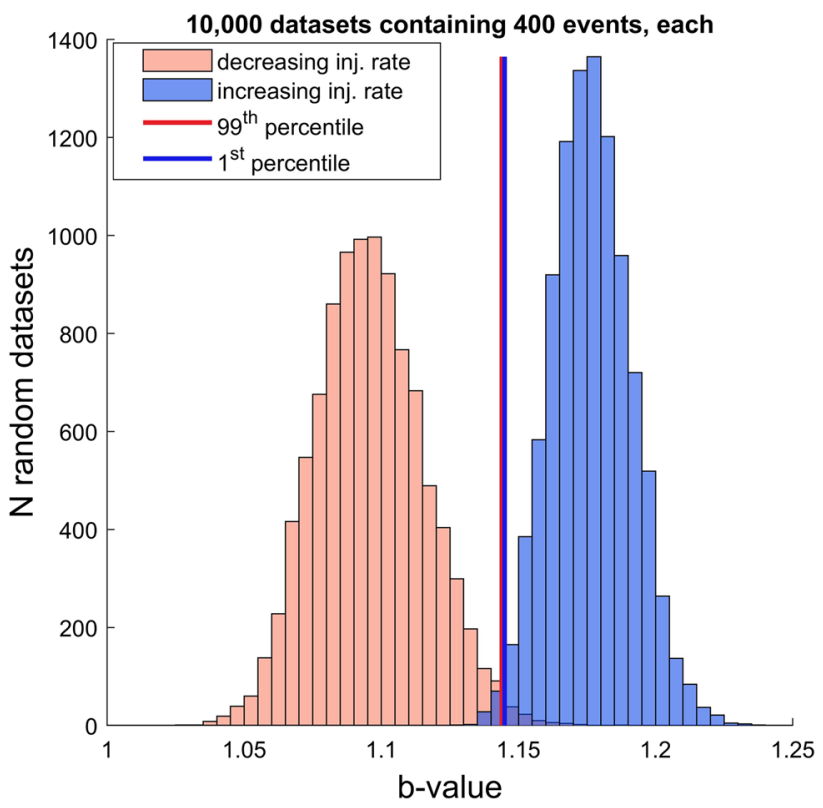

Fig. $3 b$ values calculated from 10,000 randomly selected datasets of 400 events each, corresponding to decreasing (red) or increasing (blue) injection rates. The red vertical line depicts the 99th percentile of the $b$ value obtained from the period of decreasing injection rates. The blue vertical line depicts the first percentile of the $b$ value obtained from the period of increasing injection rates
Fig. 2 Estimation of $b$ value for the 20 datasets described in the main text. Blue-shaded area corresponds to periods of increasing injection rate, red-shaded area corresponds to periods of decreasing injection rate, and gray-shaded area corresponds to periods of almost stable injection rate. The horizontal and vertical error bars indicate one bootstrap standard deviation $(\sigma)$ of the injection rates' slopes and $b$ values, respectively. The slope of the least-square linear regression curve, denoted by the dashed line, is $\sim 1$

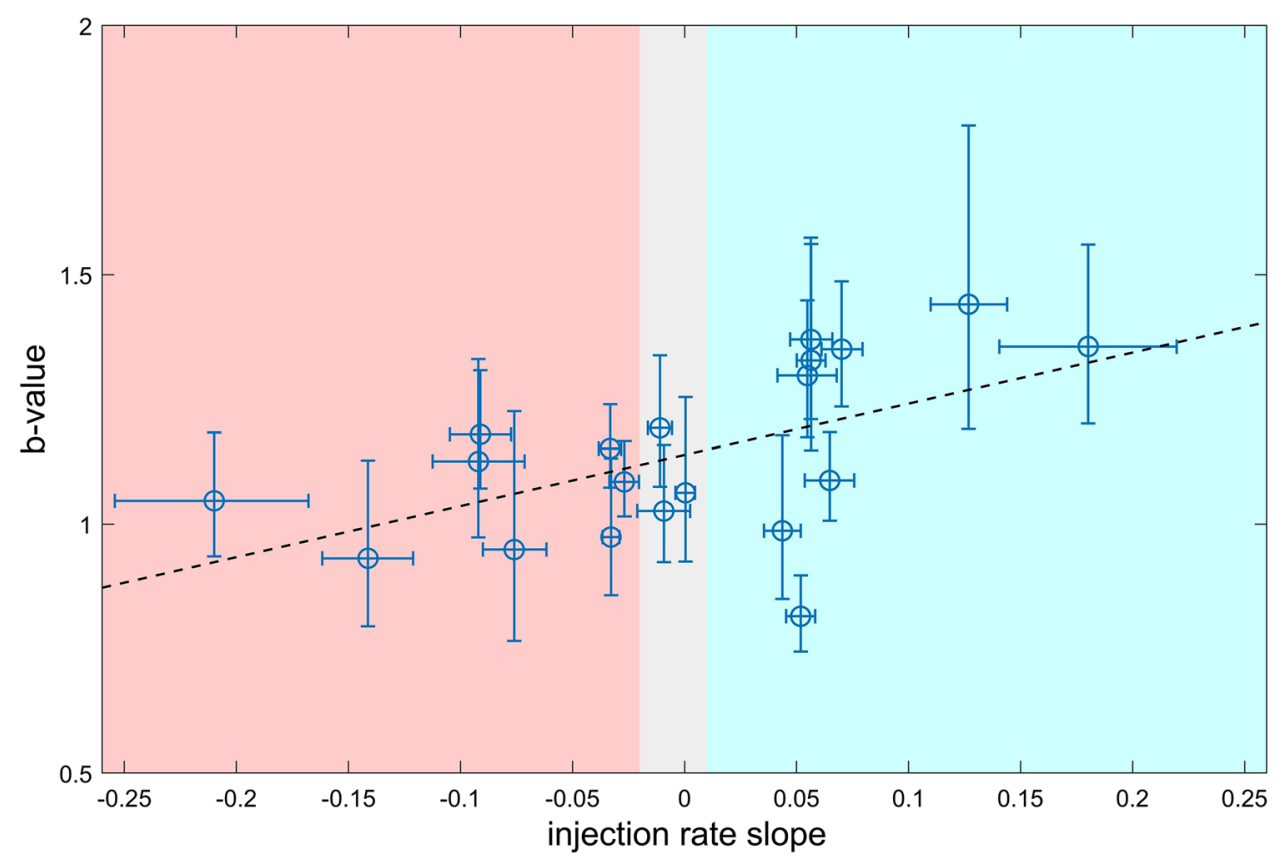


a clear difference between the $b$ values corresponding to the two events families. 10,000 bootstrap resampling trials of 400 -event subsets randomly drawn from the original datasets were performed in order to constrain the $99 \%$ confidence interval of the $b$ value estimation. As shown in Fig. 3, the bootstrap resampling $b$ value estimation histogram of the two events families roughly overlaps, indicating that the corresponding $b$ values of the increasing and decreasing injection rate periods are essentially different. The seismicity which occurred during periods of increasing injection rate demonstrates $b=1.18 \pm 0.06$, whereas the seismicity which occurred during periods of decreasing injection rate has a lower $b=1.10 \pm 0.05$. More important is the fact that the aforementioned values are differ statistically with each other at 0.01 significance level, which strongly indicates a direct influence of injection rate slope on the induced events magnitude distribution.

The next step was to apply the repeated medians technique to estimate the $b$ values ( $b^{\mathrm{RM}}$ ) for the two periods of increasing and decreasing injection rate and to quantify the significance of their difference. For this purpose, the datasets which were described in the previous paragraph were considered. The obtained results, although differing considerably from the corresponding ones derived by the maximum likelihood $b$ value estimate, still indicate a significant difference in the magnitude distribution of the two datasets: $b_{\text {increasing }}^{\mathrm{RM}}=1.97\left(S E_{\text {increasing }}=0.20\right)$ and $b_{\text {decreasing }}^{\mathrm{RM}}=1.50$ $\left(S E_{\text {decreasing }}=0.13\right)$ are both higher than the maximum likelihood estimates; however, they still indicate an equivalent difference in the magnitude distribution between the two periods. The value of the $t$-statistic (Eq. 3) was found to equal 1.98 indicating that the $b$ values of the two datasets differ from each other at 0.05 significance level. Note that due to the fact that we are interested in variation of magnitude distribution rather than the absolute $b$ values, both techniques imply a significant change of the proportion of stronger to weaker events during periods of increasing and decreasing injection rate. Figure 10 in appendix shows the relative frequency of events magnitudes during the two periods.

Subsequently, we investigated a potential delay of magnitude distribution response to fluid injection operations. The degree of the delay mainly depends on fluid diffusivity, distance from the injection well, in situ stresses, rock properties, and preexisting fractures (Goebel et al. 2015; Langenbruch and Zoback 2016). Leptokaropoulos et al. (2018b) demonstrated that the acceptable time delay between significant seismicity rate changes and fluid injection variations at 0.01 significance level lies between 0 and 80 days. They found a maximum Pearson correlation coefficient corresponding to 15-day time lag. Based on these results, we seek for significant correlation between injection rates slopes and $b$ values obtained from seismicity exhibiting a time delay equal to $0-80$ days. This was done by considering the total injection rate slope values in the 20 time periods described earlier and the seismicity which occurred in time windows shifted by the previously mentioned time lag values (0-80 days). This cross-correlation approach was quantified
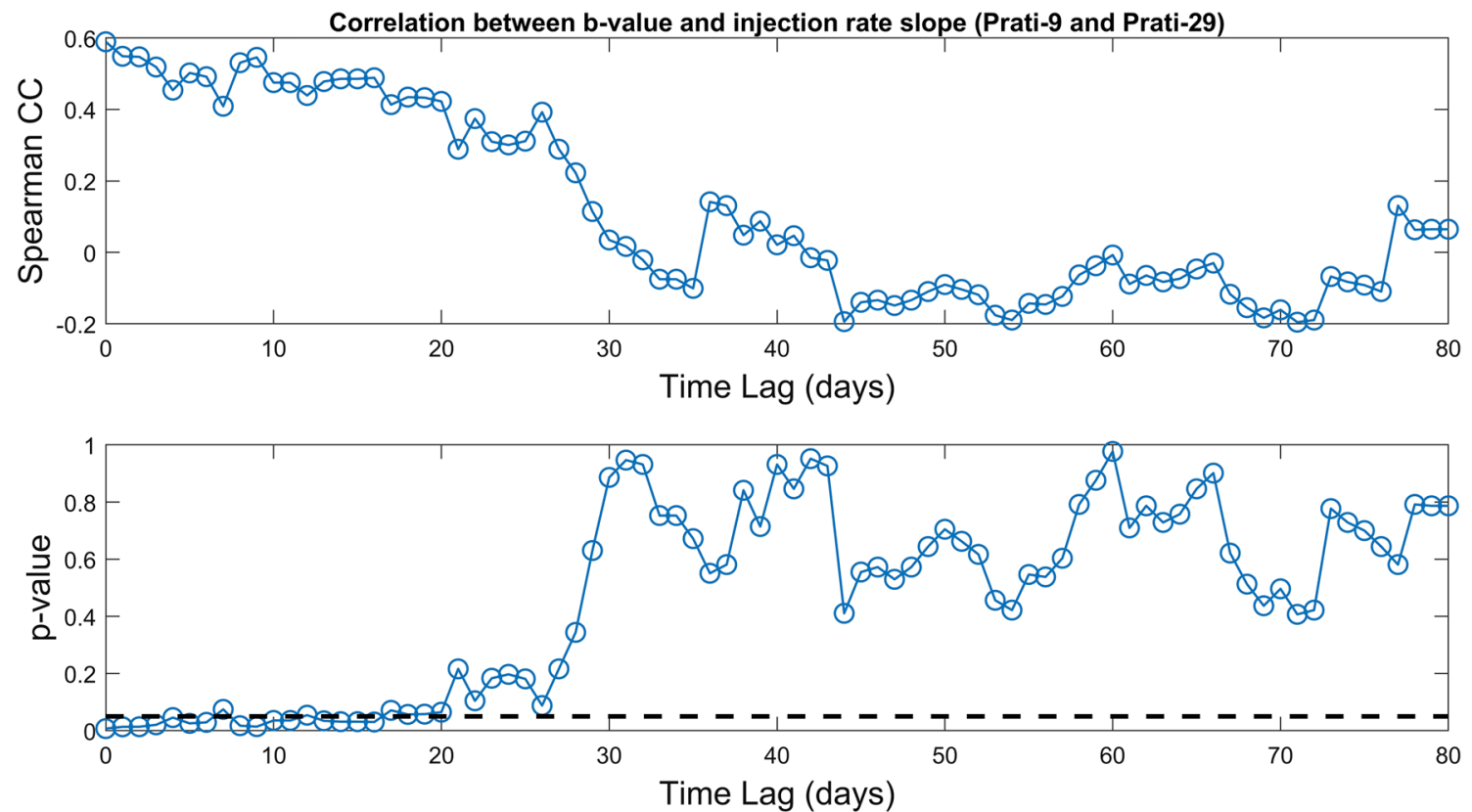

Fig. 4 SCC between average total injection rate variation (slope) and $b$ values for the 20 study periods, considering time lags from 0 to 80 days (upper frame). The corresponding $p$ values for testing the null hypothesis that the obtained correlation could result by chance are shown in the lower frame. The dashed horizontal line indicates the 0.05 significance level boundary 
by the evaluation of the SCC between the $b$ values and the injection rates slopes time series. The corresponding $p$ values indicating the significance of the null hypothesis that a particular SCC value could be obtained at random were also calculated. These results are shown in Fig. 4 and indicate that for most of time lags between 0 and 16 days (with exception of lag $=7$ days and lag $=12$ days), the positive correlation between injection rate changes and $b$ values is statistically significant at 0.05 level. SCC for these cases is found in the range between 0.46 and 0.59 . Moreover, all time lags larger than 20 days are not statistically significant at 0.10 level. The three lowest $p$ values correspond to time lags equal to 0,1 , and 2 days, respectively. These facts indicate a prompt and direct response of the induced seismicity magnitude distribution to the injection rate variation.

Up to this point the total pumping rate from both injection wells Prati-9 and Prati-29 was considered (see Data section for details). Here, the individual contribution of Prati-9 which is located in the close vicinity of the main seismicity cloud is considered. Significance of correlation between $b$ values and injection rate variations as well as the potential delayed effect are also studied in this case. The results are demonstrated in Fig. 5, and they are similar to those shown in Fig. 4. The lowest $p$ value corresponds to zero time lag as well, whereas for the most of time lags between 0 and 9 days (with exception of lag $=4$ days and lag $=7$ days), the positive correlation between injection rate changes and $b$ values is statistically significant at 0.05 level. SCC for these cases is found in the range between 0.46 and 0.51 , slightly lower than the SCC values obtained by considering the influence of both injection wells. This means that although both wells contribute to $b$ values, the contribution of Prati-9 is much more significant, what makes absolute sense considering the location of its open hole, almost in the center of seismicity cloud (Fig. 1a).

An additional approach to be tested regards the connection between $b$ values and absolute values of injection rate. For this purpose, new time periods were specified corresponding to fluid injection rates between predefined levels. This approach was followed in order to investigate whether higher or lower injection rates lead to significant changes in the $b$ values of the seismic activity. For injection rate bins of $2000 \mathrm{~m}^{3} /$ day (Table 1) and for injection rate bins of $3000 \mathrm{~m}^{3} /$ day (Fig. 6), no unequivocal conclusions could be made. No particular pattern or correlation can be found between magnitude distribution and absolute values of injection rates. This is possibly because of the continuously and rapidly changing injection rates throughout the study period which makes it difficult to detect a possible influence of total injection rate on $b$ values. On the other hand, as shown in the previous analysis, $b$ values can be directly associated with injection rate slope variations.

We further examined a potential association between $b$ values fluctuation and the spatial distribution of the microseismicity as well as with the corresponding static stress drops. The $b$ values fluctuation in connection to the events distribution was partially showed in Leptokaropoulos et al. (2018b). It was showed there that the differences of $b$ values
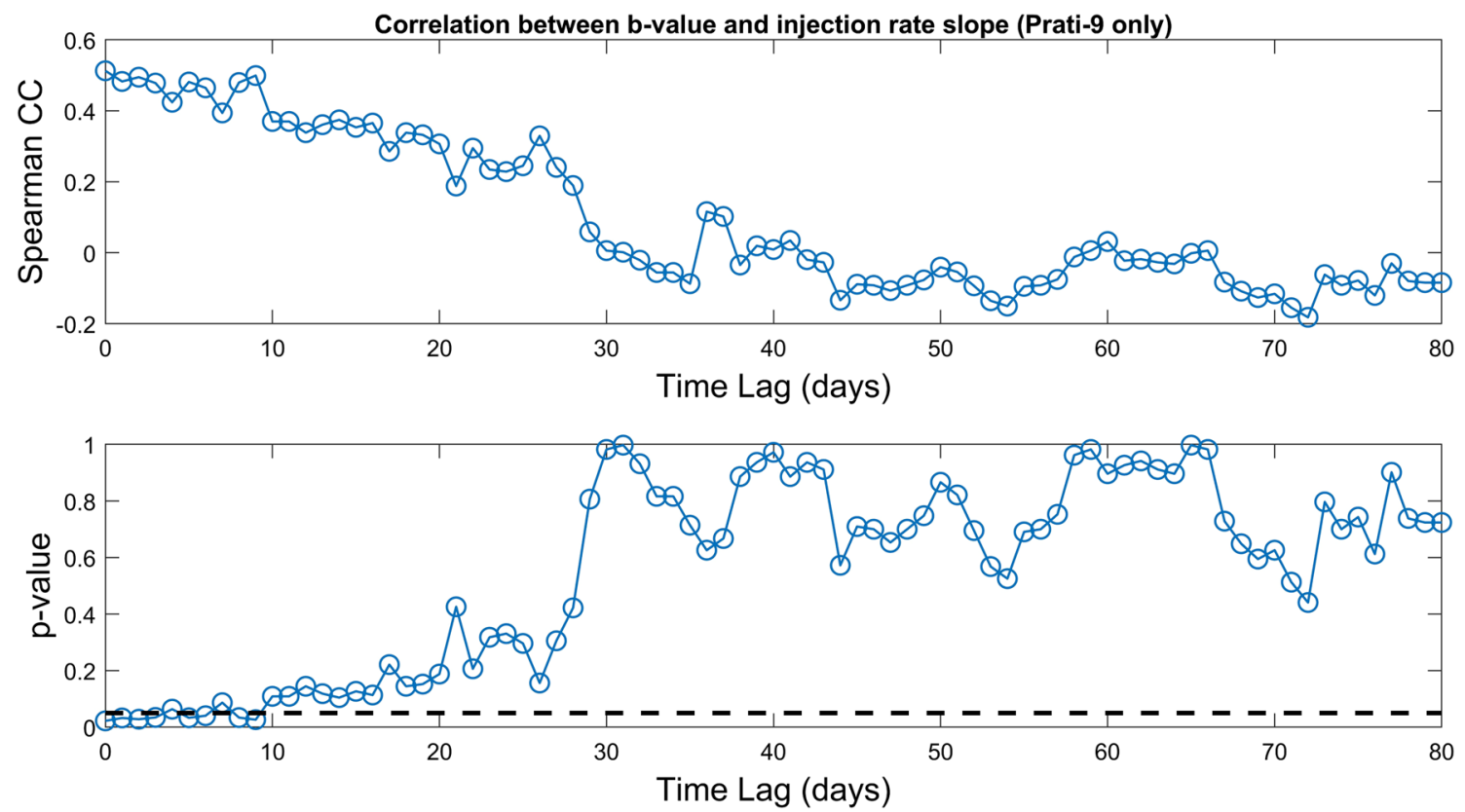

Fig. 5 SCC between average injection rate variation (slope) in Prati-9 and $b$ values for the 20 study periods, considering time lags from 0 to 80 days (upper frame). The corresponding $p$ values for testing the null hypothesis that the obtained correlation could result by chance are shown in the lower frame. The dashed horizontal line indicates the 0.05 significance level boundary 
Table 1 Estimated $b$ values (column 4) for datasets corresponding to selected values of absolute total injection rate (column 1)

\begin{tabular}{lrlllcl}
\hline Injection rate $\left(\mathrm{m}^{3} /\right.$ day $)$ & $\mathrm{N}$ & Mean depth $(\mathrm{m})$ & $b$ value & $b$ value within one $\sigma$ & $N$ (for SD) & $\log _{10}(\mathrm{SD})$ \\
\hline $0-2000$ & 130 & $2517 \pm 183$ & 1.20 & {$[1.11-1.31]$} & 19 & $6.62 \pm 0.36$ \\
$2000-4000$ & 241 & $2490 \pm 176$ & 1.06 & {$[1.00-1.12]$} & 54 & $6.78 \pm 0.34$ \\
$4000-6000$ & 316 & $2471 \pm 180$ & 1.17 & {$[1.11-1.23]$} & 100 & $6.68 \pm 0.35$ \\
$6000-8000$ & 270 & $2458 \pm 198$ & 1.12 & {$[1.07-1.18]$} & 76 & $6.76 \pm 0.32$ \\
$8000-10,000$ & 53 & $2459 \pm 186$ & 1.26 & {$[1.12-1.42]$} & 22 & $6.70 \pm 0.33$ \\
$10,000-12,000$ & 111 & $2428 \pm 191$ & 1.18 & {$[1.10-1.28]$} & 45 & $6.66 \pm 0.35$ \\
\hline
\end{tabular}

The standard deviation $(\sigma)$ of the $b$ value was obtained by bootstrap resampling and shown in column 5 . The number of events, $\mathrm{N}$, in each dataset for depth and $b$ value calculations is shown in column 2 . The mean depth of the events and its $\sigma$ is shown in the column 3. The mean decimal logarithm of static stress drop and its $\sigma$ is shown in column 7, whereas column 6 shows the number of events used for stress drop statistics

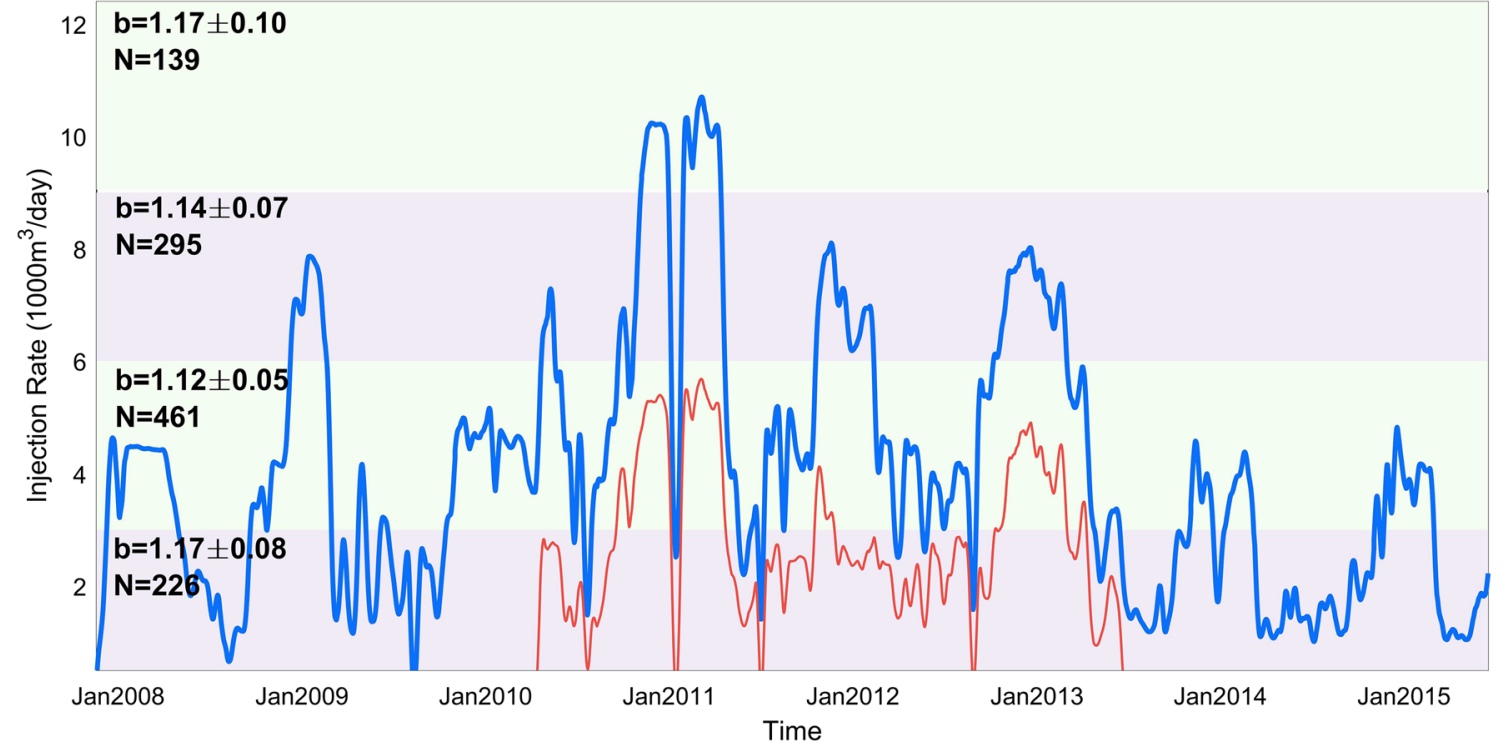

Fig. $6 b$ values (shown on the left side) calculated for specified ranges of absolute total injection rate values (blue curve). From top to bottom: $0-3,000 ; 3000-6000 ; 6000-9000 ; 9000-12,000 \mathrm{~m}^{3} /$ day. The red curve shows the partial contribution of Prati-29 in the total fluid injected. Note that when the red curve is equal to zero, the blue curve represents the fluid injected in Prati-9. The $b$ values standard deviations are estimated by Eq. 2 with distance from the injection well are small and not systematic for indicating some connection (Table 2 from Leptokaropoulos et al. 2018b). Concerning the depth distribution of the $b$ values, Fig. 7 shows a slight trend of decreasing $b$ value toward deeper layers. The plot was obtained by sorting the events by depth and applying a moving window of 100 events, sliding by 1 event. It is shown that although the shallowest segments demonstrate larger $b$ values than the deepest ones, there are significant fluctuations at the layers in-between. When we performed correlation analysis for 11 non-overlapping datasets of 100 events each, we obtained a SCC $=0.28$ with $p=0.40$, and therefore, this trend is not significant. The depth $/ b$ value distribution of the events within each of the periods with increasing injection rate and decreasing injection rate is also illustrated in Fig. 8a. As shown, no significant linear correlation is obtained, justified also by a SCC $=-0.2$ with $p=0.40$. The corresponding SCC between injection rate slope and depth of the events is equal to -0.13 with $p=0.59$. This suggests that there is no significant depth migration of the events with increasing/ decreasing injection rates. That probably occurs because of the pulsating seismicity cloud resulting from the injection fluctuation (Martínez-Garzón et al. 2014), which inflates and collapses rather symmetrically toward the horizontal and vertical directions. From these evidence, we can conclude that in our case study, the depth/distance of the events only constitutes a second-order cause (if any at all) affecting the $b$ values.

Regarding the connection between static stress drop and $b$ values, we found no statistically significant correlation 


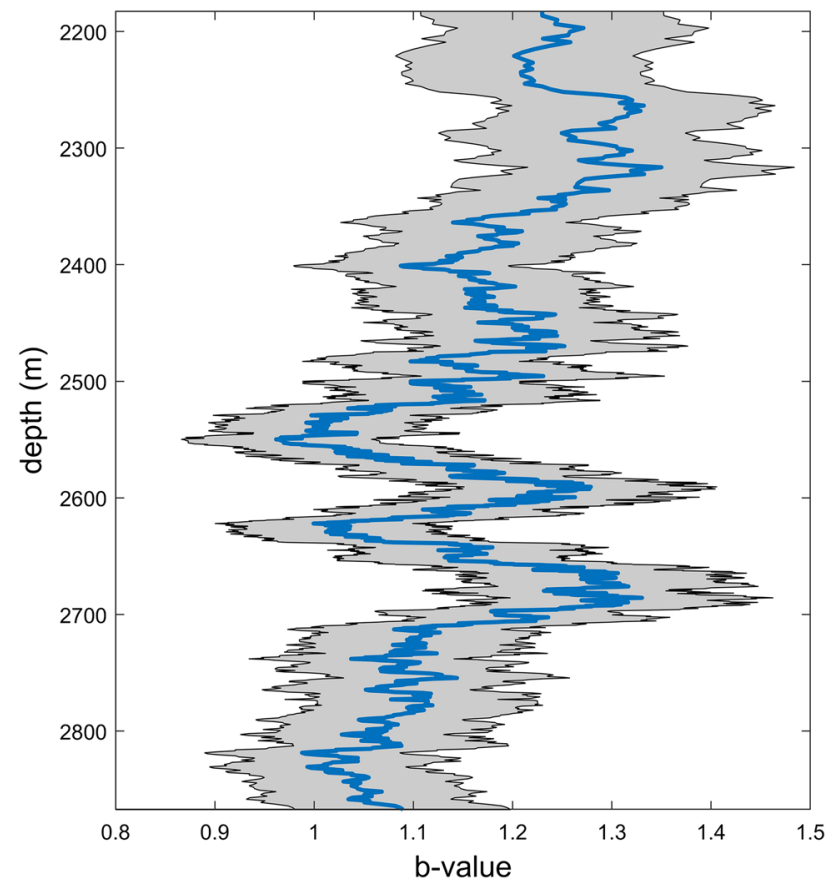

Fig. $7 b$ value distribution with the depth of the events. The shaded area indicates the bootstrap standard deviation
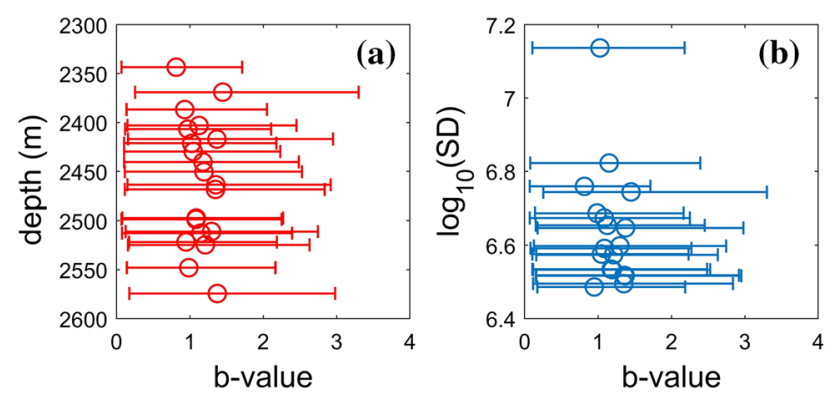

Fig. 8 Scatter plot between $b$ value and mean depth of the events included in each one of the 20 periods shown in Fig. 2. a Scatter plot between $b$ value and mean stress drop values of the events included in 15 of the periods shown in Fig. 2. For the remaining five periods before February 1, 2010, there are no calculated stress drop values. b The error bars indicate one bootstrap standard deviation of the $b$ value

between these two parameters (Fig. 8b). For the 15 out of 20 distinguished periods, where data are available, the SCC between $b$ values and mean decimal logarithm of stress drops is -0.08 , with a corresponding $p=0.77$. The values of mean static stress drop and mean depth shown in Table 1 also do not show any clear relation with neither $b$ values, nor absolute injection rate values. Although Staszek et al. (2017) showed that there is an inverse relation between stress drops and absolute injection rates, this is a long-term relation, which can be seen in relatively large samples and long time periods, and therefore, it cannot be noticed in our analysis. Our correlation analysis clearly demonstrates that the injection rate slope effect is dominant in comparison with the depth distribution and stress drop.

Summarizing, the following significant results have been obtained from our analysis:

- Positive linear correlation at 0.01 significance level, between injection rate slope and $b$ values (no time lag applied).

- Statistically significant difference, between $b$ values of events occurred during increasing and during decreasing injection rates (at 0.01 level when $b$ values were estimated by maximum likelihood and at 0.05 level when $b$ values were estimated by the repeated medians technique).

- Positive linear correlation at 0.05 significance level, between injection rate slope and $b$ values, considering both injection wells, for time lags equal to $0-16$ days (with exception for time lags equal to 7 and 12 days, where $p$ is slightly higher).

- Positive linear correlation at 0.05 significance level, between injection rate slope and $b$ values, considering only Prati-9 injection well, for time lags equal to 0-9 days (with exception for time lags equal to 4 and 7 days, where $p$ is slightly higher).

\section{Discussion and conclusions}

In The Geysers area, injection-induced thermal stresses (near field) and pore pressure changes (near and far field) were proved to be responsible for the seismicity occurrence (Martínez-Garzón et al. 2014; Rutqvist et al. 2013). The results presented above indicate that the volume of the injected fluid increases pore pressure as well as the G-R law $b$ values. This is in agreement with both theoretical concept and field observations. The positive relationship between $b$ value and pore pressure has been suggested previously by Bachmann et al. $(2011,2012)$ at the Deep Heat Mining project in Basel, Switzerland. They observed a substantial decrease in $b$ value during post-injection period ( $b$ value difference between co- and post-injection periods equaled 0.43 according to Bachmann et al. 2011) and a decrease in $b$ value with increasing distance from the injection well (Bachmann et al. 2012). On the other hand, Leptokaropoulos et al. (2018b) did not come up with a significant $b$ value reduction with distance from Prati-9 injection well, probably due to cyclic character of injection and pulsation of seismicity cloud in The Geysers area (Martínez-Garzón et al. 2014; Kwiatek et al. 2015). An observation of high $b$ values connected with high injection rates was also made at Soultz-sous-Forets GPK2 well by 
Stormo et al. (2015). It must be stated, however, that in some cases anomalously low $b$ value during peak injection intervals were reported (e.g., Martínez-Garzón et al. 2014; Goebel et al. 2015; Huang and Beroza 2015). In the analyzed part of The Geysers geothermal field, Kwiatek et al. (2015) did not observe any long-term $b$ value trend; however, they reported its short-term temporal changes. In addition, a temporal $b$ value analysis shows that during the first cycles of injection, the $b$ values are remarkably lower, and then they fluctuate between higher values.

Although the statistical analysis definitely reveals a positive relation between injection rate slope and $b$ values, the complexity of the underlying physical mechanisms and the data limitations do not allow for a robust interpretation of the results. The fact that $b$ value is found to be related to injection rate slope, not absolute injection rate values, could suggest a dynamic reservoir response to increasing pore pressure, which is changing over time. During the first increasing injection rate periods, stress accumulated on pre-stressed tectonic faults was released by events triggered by a sudden increase in pore pressure (considerably lower $b$ values, Appendix Fig. 11). Then, repeated injection cycles might lead to the opening of new fractures (induced events), especially after the start of injection into Prati-29 well (Fig. 11). This thesis has been confirmed by Martínez-Garzón et al. (2017) who observed increased content of non-double couple components of earthquake mechanisms during the peak injection periods. However, fractures generated during each subsequent increasing injection period remained open, so the reservoir structure was becoming more and more cracked with time, generating new pathways for the injected fluid. Therefore, the same values of injection rate would generate lower pore pressure during later injection peaks leading to higher effective normal stress on the faults and lower $b$ values (Fig. 11). In addition, injecting water at decreasing rates may produce an effect similar to the $b$ value decrease after the shut-in phase (e.g., Bachmann et al. 2012). However, due to the limited data and some potential second-order phenomena (possible time delay of the diffusion process, thermal effects, co-seismic stresses, etc.), there are some deviations in the calculated $b$ values. Nevertheless, we managed to established a statistical significant relationship between the slope of injection rate and $b$ values. Future studies oriented toward this direction utilizing more data may reveal more information on a possible physical interpretation mechanism.

From the results of our analysis we may conclude to the following points concerning the analyzed area:

- The G-R law $b$ value is proportional to the slope of the injection rate (second derivative of injection volume), but not to the absolute injection rate values.

- The magnitude distribution of the induced events follows immediately or shortly after (0-15-day time lag) the injection rate changes.

- Prati-9 injection well, which is located in the close vicinity of the seismicity cloud, contributes mainly, but not exclusively to the $b$ value fluctuation.

- A relation between static stress drops/vertical distribution of microseismicity and $b$ values could not be identified under the current analysis.

Acknowledgments This work was supported within SHEER: 'SHale gas Exploration and Exploitation induced Risks' project funded from the European Union Horizon 2020-Research and Innovation Programme, under grant agreement 640896 (www.sheerproject.eu). The work was also partially supported within statutory activities No 3841/E-41/S/2018 of the Ministry of Science and Higher Education of Poland. The data used in the paper are available in IS-EPOS e-Platform of Thematic Core Service-Anthropogenic Hazards (TCS-AH) of EPOS (Kwiatek et al. 2015; Martínez-Garzón et al. 2014; IS EPOS 2017, Episode: THE GEYSERS Prati 9 and Prati 29 cluster, https://tcs.ah-epos. eu/\#episode:THE_GEYSERS_Prati_9_and_Prati_29_cluster, https:// doi.org/10.25171/instgeoph_pas_isepos-2017-011).

Open Access This article is distributed under the terms of the Creative Commons Attribution 4.0 International License (http://creativeco mmons.org/licenses/by/4.0/), which permits unrestricted use, distribution, and reproduction in any medium, provided you give appropriate credit to the original author(s) and the source, provide a link to the Creative Commons license, and indicate if changes were made.

\section{Appendix}

\section{Data outlier}

Figure 9. 
Fig. 9 Estimation of $b$ value for the 21 datasets described in the main text. Notification as in Fig. 2. The outlier is indicated by red circle and red error bars and was removed before proceeding to further analysis

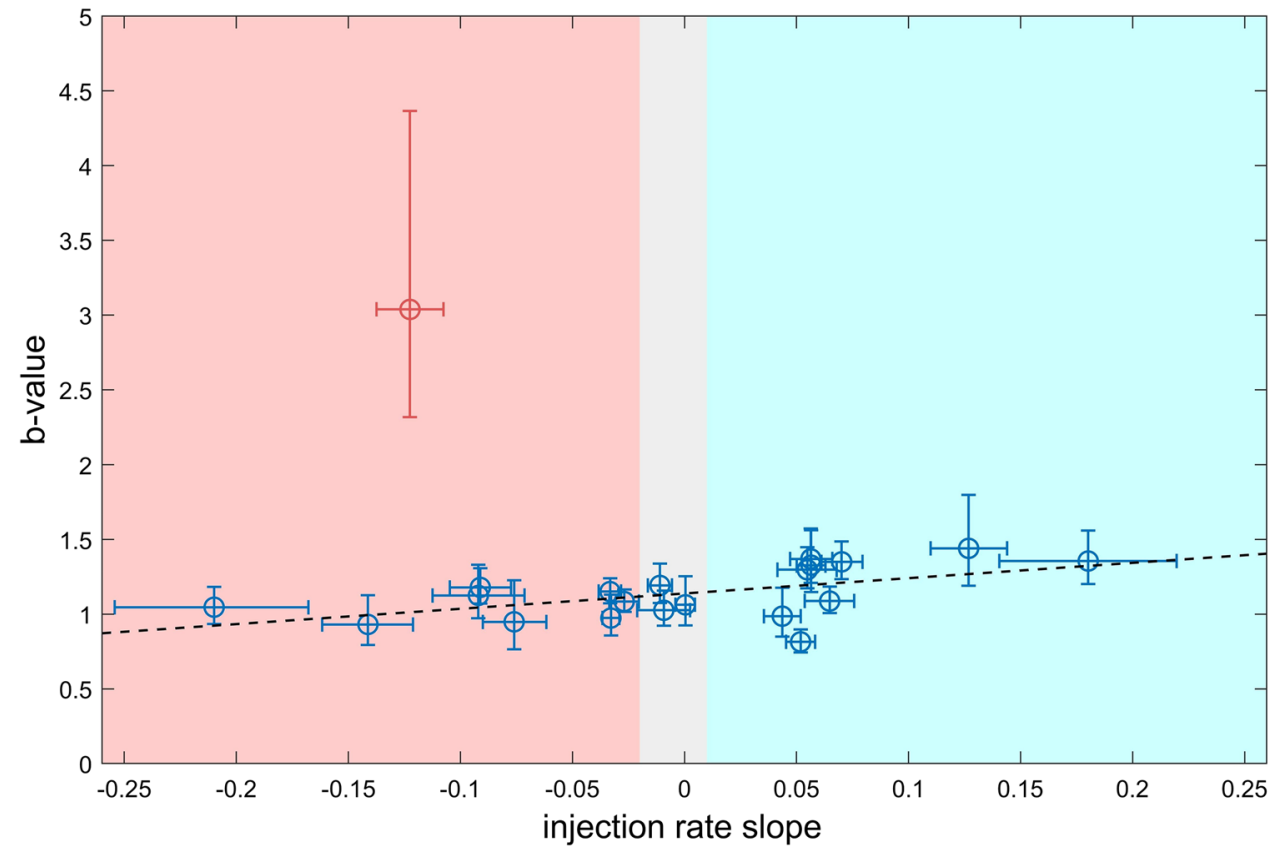

\section{Relative frequency of magnitudes}

Figure 10.

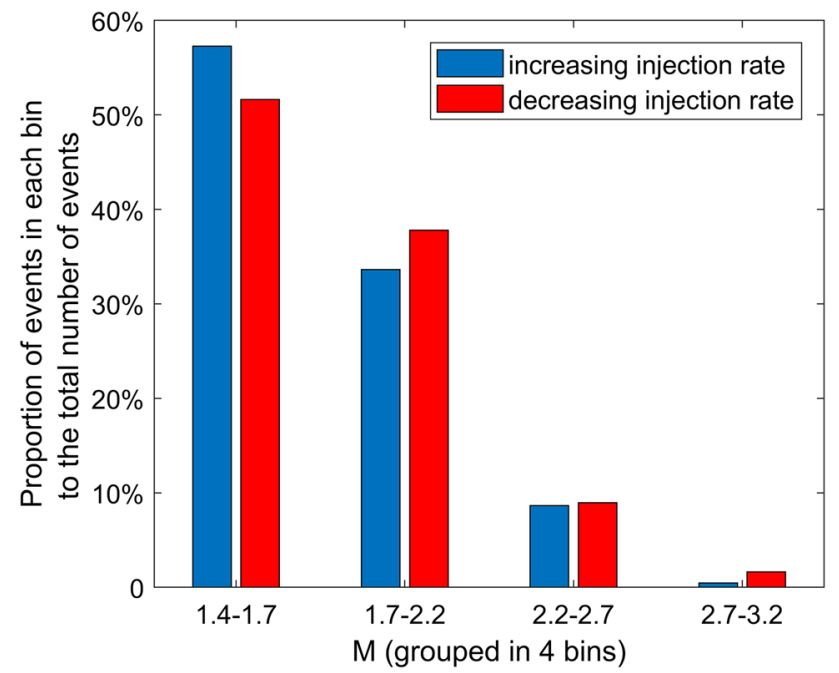

Fig. 10 Relative frequency (percentage) of magnitude distribution for the two datasets corresponding to increasing (blue) and decreasing (red) injection rates. The magnitudes have been grouped in four classes (bins) of weakest, weak, strong, and strongest events. The decreasing injection rate periods include larger proportion of stronger than weaker events in comparison with the periods of increasing injection rate

\section{$b$ values in time}

Figure 11.

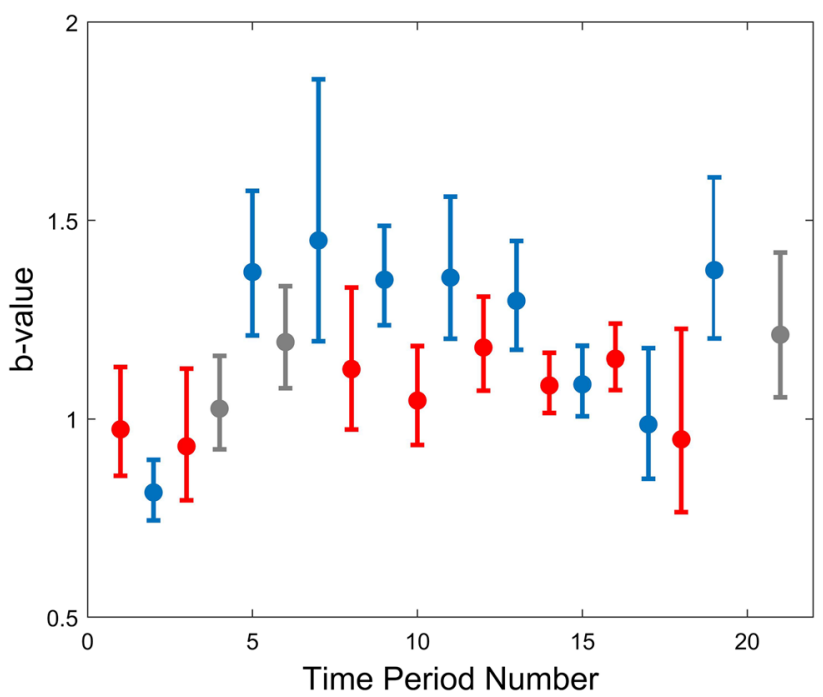

Fig. $11 b$ values with bootstrap standard deviation estimated for the 21 defined time periods. $b$ value for period number 20 is out of scale of the figure. Increasing, decreasing, and stable injection rate periods are indicated with blue, red, and gray error bars, respectively 


\section{References}

Aki K (1965) Maximum likelihood estimate of $b$ in the formula $\log N=a-\mathrm{bM}$ and its confidence limits. Bull Earthq Res Inst Tokyo Univ 43:237-239

Amorèse D, Grasso J-R, Rydelek PA (2010) On varying $b$ values with depth: results from computer-intensive tests for Southern California. Geophys J Int 180:347-360. https://doi.org/10.1111/j.1365246X.2009.04414.X

Bachmann C, Wiemer S, Woessner J, Hainzl S (2011) Statistical analysis of the induced basel 2006 earthquake sequence: introducing a probability-based monitoring approach for enhanced geothermal systems. Geophys J Int 186:793-807. https://doi.org/10.1111/ j.1365-246X.2011.05068.x

Bachmann C, Wiemer S, Goertz-Allmann BP, Woessner J (2012) Influence of pore-pressure on the event-size distribution of induced earthquakes. Geophys Res Lett 39:L09302. https://doi. org/10.1029/2012GL051480

Bender B (1983) Maximum likelihood estimation of $b$ values for magnitude grouped data. Bull Seismol Soc Am 73:831-851

Davies R, Foulger G, Bindley A, Styles P (2013) Induced seismicity and hydraulic fracturing for the recovery of hydrocarbons. Mar Pet Geol 45:171-185

Dempsey D, Suckale J, Huang Y (2016) Collective properties of injection0induced earthquake sequences: 2. Spatiotemporal evolution and magnitude frequency distributions. J Geophys Res Solid Earth. https://doi.org/10.1002/2015JB012551

Edwards B, Douglas J (2014) Magnitude scaling of induced earthquakes. Geothermics 52:132-139

El-Isa ZH, Eaton DW (2014) Spatiotemporal variations in the $b$ value of earthquake magnitude-frequency distributions: classification and causes. Tectonophysics 615-616:1-11

Evans KF, Zappone A, Kraft T, Deichmann N, Moia F (2012) A survey of the induced seismic responses to fluid injection in geothermal and $\mathrm{CO}_{2}$ reservoirs in Europe. Geothermics 41:30-54

Goebel THW, Hauksson E, Aminzadeh F, Ampuero J-P (2015) An objective method for the assessment of fluid injection-induced seismicity and application to tectonically active regions in central California. J Geophys Res Solid Earth. https://doi. org/10.1002/2015JB011895

Goebel THW, Hosseini SM, Cappa F, Hauksson E, Ampuero JP, Aminzadeh F, Saleeby JB (2016) Wastewater disposal and earthquake swarm activity at the southern end of the Central Valley, California. Geophys Res Lett. https://doi.org/10.1002/2015GL066948

Goertz-Allmann BP, Wiemer S (2013) Geomechanical modeling of induced seismicity source parameters and implications for seismic hazard assessment. Geophysics. https://doi.org/10.1190/GEO20 12-0102.1

Grigoli F, Cesca S, Priolo E, Rinaldi AP, Clinton JF, Stabile TA, Dost B, Fernandez MG, Wiemer S, Dahm T (2017) Current challenges in monitoring, discrimination, and management of induced seismicity related to underground industrial activities: a European per- spective. Rev Geophys 55:310-340. https://doi. org/10.1002/2016RG000542

Hillis RR (2003) Pore pressure/stress coupling and its implications for rock failure. In: Subsurface sediment mobilization, 216, Geological Society of London, pp 359-368. Special Publication

Huang Y, Beroza G (2015) Temporal variation in the magnitudefrequency distribution during the Guy-Greebrier earthquake sequence. Geophys Res Lett 42:6639-6646. https://doi. org/10.1002/2015GL065170

IS EPOS (2017) Episode: The Geysers Prati 9 and Prati 29 cluster, https://tcs.ah-epos.eu/\#episode:THE_GEYSERS_Prati_9_and_ Prati_29_cluster, https://doi.org/10.25171/instgeoph_pas_isepo s-2017-011
Kijko A, Sellevoll MA (1989) Estimation of earthquake hazard parameters from incomplete data files. Part I. Utilization of extreme and complete catalogs with different threshold magnitudes. Bull Seismol Soc Am 79:645-654

Kwiatek G, Martínez-Garzón P, Dresen G, Bohnhoff M, Sone H, Hartline C (2015) Effects of long-term fluid injection on induced seismicity parameters and maximum magnitude in northwestern part of The Geysers geothermal field. J Geophys Res Solid Earth 120:7085-7101. https://doi.org/10.1002/2015JB012362

Langenbruch C, Zoback MD (2016) How will induced seismicity in Oklahoma respond to decreased saltwater injection rates? Sci Adv. https://doi.org/10.1126/sciadv.1601542

Leptokaropoulos K, Staszek M, Cielesta S, Olszewska D, Urban P, Lizurek G (2017) Time dependent seismic hazard in Bobrek coal mine, Poland, assuming different magnitude distribution estimations. Acta Geophys. https://doi.org/10.1007/s11600-016-0002-9

Leptokaropoulos K, Adamaki A, Roberts R, Gkarlaouni C, Paradisopoulou $\mathrm{P}$ (2018a) Impact of magnitude uncertainties on seismic catalog properties. Geophys J Int. https://doi.org/10.1093/gji/ ggy023

Leptokaropoulos K, Staszek M, Lasocki S, Martínez-Garzón P, Kwiatek G (2018b) Evolution of seismicity in relation to fluid injection in North-Western part of The Geysers geothermal field. Geophys J Int 212:1157-1166. https://doi.org/10.1093/gji/ggx481

López-Comino JA, Cesca S, Jarosławski J, Montcoudiol N, Heimann S, Dahm T, Lasocki S, Gunning A, Capuano P, Ellsworth WL (2018) Induced seismicity response of hydraulic fracturing: results of a multidisciplinary monitoring at the Wysin site, Poland. Sci Rep 5:2. https://doi.org/10.1038/s41598-018-26970-9

Majer EL, Peterson JE (2007) The impact of injection on seismicity at The Geysers, California geothermal field. Int J Rock Mech Min Sci 44(8):1079-1090. https://doi.org/10.1016/j.ijrmm s.2007.07.023

Majer EL, Baria R, Stark M, Oates S, Bommer J, Smith B, Asanuma H (2007) Induced seismicity associated with enhanced geothermal Systems. Geothermics 36:185-222

Martínez-Garzón P, Kwiatek G, Sone H, Bohnhoff M, Dresen G, Hartline C (2014) Spatiotemporal changes, faulting regimes, and source parameters of induced seismicity: a case study from The Geysers geothermal field. J Geophys Res Solid Earth 119:83788396. https://doi.org/10.1002/2014JB011385

Martínez-Garzón P, Kwiatek G, Bohnhoff M, Dresen G (2017) Volumetric components in the eq source related to fluid injection and stress state. Geophys Res Lett 44:800-809. https://doi. org/10.1002/2016GL071963

Martínez-Garzón P, Zaliapin I, Ben-Zion Y, Kwiatek G, Bohnhoff M (2018) Comparative study of earthquake clustering in retlation to hydraulic activities at geothermal fileds in California. J Geophys Res. https://doi.org/10.1029/2017JB014972

Maxwell SC, Jones M, Parker R, Miong S, Leaney S, Dorval D, D'Amico D, Logel J, Anderson E, Hammermaster K (2009) Fault activation during hydraulic fracturing. In: Proceedings of 79th SEG annual meeting, Huston, Texas, 1552-1556

Oppenheimer DH (1986) Extensional tectonics at The Geysers Geothermal Area, California. J Geophys Res. https://doi.org/10.1029/ JB091iB11p11463

Rutqvist J, Dobson PF, Garcia J, Hartline C, Jeanne P, Oldenburg CM, Vasco DW, Walters M (2013) The Northwest Geysers EGS Demonstration Project, California: pre-stimulation modeling and interpretation of the stimulation. Math Geosci. https://doi.org/10.1007/ s11004-013-9493-y

Schoenball M, Müller T, Müller B, Heidbach O (2010) Fluid-induced microseismicity in pre-stressed rock masses. Geophys J Int 180:813-819. https://doi.org/10.1111/gji.2010.180.issue-2 
Scholz C (2015) On the stress dependence of the earthquake b value. Geophys Res Lett. https://doi.org/10.1002/2014GL062863

Siegel AF (1982) Robust regression using repeated medians. Biometrika 69:242-244

Smirnov MY (2003) Magnetotelluric data processing with a robust statistical procedure having a high breakdown point. Geophys J Int. https://doi.org/10.1046/j.1365-246X.2003.01733.x

Staszek M, Orlecka-Sikora B, Leptokaropoulos K, Martínez-Garzón P, Kwiatek G (2017) Temporal static stress drop variations in relation to technological activity at The Geysers geothermal field, California. Geophys Res Lett. https://doi.org/10.1002/2017GL073929

Stormo A, Lengliné O, Schmittbuhl J (2015) Mechanical origin of $b$ value changes during stimulation of deep geothermal reservoirs. Geotherm Energy. https://doi.org/10.1186/s40517-014-0022-0

Terzaghi K (1943) Theoretical soil mechanics. Wiley, New York, p 510
Urban P, Lasocki S, Blascheck P, Do Nascimento AF, Giang NV, Kwiatek G (2016) Violations of Gutenberg-Richter in anthropogenic seismicity. Pure appl Geophys 173:1517-1537

Utsu T (1999) Representation and analysis of the earthquake size distribution: a historical review and some new approaches. Pure appl Geophys 155:509-535

Wiemer S, Wyss M (2000) Minimum magnitude of completeness in earthquake catalogs: examples from Alaska, the Western United States, and Japan. Bull Seismol Soc Am 90(4):859-869

Wyss M (1972) Towards a physical understanding of the earthquake frequency distribution. Geophys J R Astron Soc 31:341-359. https ://doi.org/10.1111/j.1365-246X.1973.tb06506.x

Zang A, Oye V, Jousset P, Deichmann N, Gritto R, McGarr A, Majer E, Bruhn D (2014) Analysis of induced seismicity in geothermal reservoirs: an overview. Geothermics 52:6-21 